\title{
La machine à dire beaucoup : mécanique du fait divers chez Valère Novarina
}

"La machine à dire beaucoup": Crime News Item's Mechanism in Valère Novarina's Writings

Sibylle Orlandi

\section{OpenEdition}

Journals

Édition électronique

URL : http://journals.openedition.org/recherchestravaux/967

ISSN : 1969-6434

Éditeur

UGA Éditions/Université Grenoble Alpes

Édition imprimée

ISBN : 978-2-37747-056-3

ISSN : 0151-1874

Référence électronique

Sibylle Orlandi, «La machine à dire beaucoup : mécanique du fait divers chez Valère Novarina »,

Recherches \& Travaux [En ligne], 92 | 2018, mis en ligne le, consulté le 08 septembre 2020. URL: http:// journals.openedition.org/recherchestravaux/967

Ce document a été généré automatiquement le 8 septembre 2020

(c) Recherches \& Travaux 


\title{
La machine à dire beaucoup : mécanique du fait divers chez Valère Novarina
}

\author{
"La machine à dire beaucoup": Crime News Item's Mechanism in Valère \\ Novarina's Writings
}

Sibylle Orlandi

\begin{abstract}
LA MACHINE À DIRE BEAUCOUP.
Duglo-Derduglo-Moribu : une requête en problématique vient d'être lancée à l'encontre de

deux points parallèles de détermination rétrospectivement incapables de s'entendre pour parvenir à réussir leur rencontre à angle droit ${ }^{1}$.
\end{abstract}

Dans La Scène, pièce publiée par Valère Novarina en 2003, apparaît un personnage qui semble être un relais de la parole médiatique : il s'agit de La Machine à dire beaucoup, dont les surgissements rythment aussi L'Acte inconnu (2007) et Le Vrai Sang (2011). Son intervention est parfois secondée par d'autres machines: La Machine à dire la suite et La Machine sans savoir pourquoi, dans La Scène; La Machine à faire l'homme, dans L'Acte inconnu $^{2}$; La Machine à marcher à l'arrêt, La Machine agglutinante, La Machine à savoir pourquoi, La Machine à voir partout, etc., dans Le Vrai Sang ${ }^{3}$ - cette dernière œuvre donne à voir une multiplication proprement étourdissante des instances mécaniques. Si, en termes quantitatifs, la place qui leur est accordée varie d'une pièce à l'autre, La Machine à dire beaucoup et ses homologues occupent un rôle relativement stable : elles se présentent comme des caisses de résonance, qui accueillent et diffusent différents discours journalistiques, avec une prédilection pour les formes brèves. Le fait divers cohabite ainsi avec des promesses électorales, des bulletins météorologiques, des bulletins d'information politique, des revues de presse, etc. Dans la présentation qu'en donne le dramaturge, la ligne de partage se révèle essentiellement rhétorique: en somme, chacune de ces formes brèves se définirait moins par un contenu que par une matrice linguistique, précisément celle que le dispositif théâtral s'efforce de mettre à 
nu. Accusant certains phénomènes syntaxiques, lexicaux et rythmiques propres aux faits divers journalistiques, le dramaturge exhibe une mécanique qui semble s'autoalimenter, dans un mouvement d'amplification incontrôlable.

\section{Regards obliques sur un genre de discours}

2 Pour assurer la reconnaissance du fait divers comme tel, mais aussi sans nul doute pour jouer avec sa logique implacable, Valère Novarina s'attache facétieusement à exemplifier jusqu'à l'outrance les traits définitoires du genre. Dans l'étude qu'il consacre au fait divers, Barthes établit deux critères stables de reconnaissance: la rupture opérée par rapport à l'ordre habituel des choses, et l'immanence du récit, qui peut être reçu et compris sans aucune connaissance préalable 4 . À ces critères, Laetitia Gonon propose d'ajouter deux caractéristiques : la rapidité de consommation, liée à la périodicité de la publication, et le statut des protagonistes, lesquels sont des gens ordinaires ${ }^{5}$. Dans les termes de Marine M'Sili, le fait divers offre «la chronique des particuliers et des anonymes ${ }^{6} »$. Autant d'éléments saillants dans ce fragment livré par La Machine à dire la suite, dans La Scène :

Le corps d'un expert-comptable de quarante-sept ans a été découvert dans son congélateur à Berck-Plage : une enquête est en cours à Limoges. (S, p. 87)

De fait, le passage est doté d'une autonomie à la fois syntaxique et informationnelle : on retrouve bien le «micro-drame » évoqué par Marine M'Sili ${ }^{7}$ : s'il est vrai que l'enquête est présentée comme en cours, il n'en demeure pas moins que l'événement marquant, celui qui constitue en quelque sorte le noyau du fait divers, est déjà livré - il s'agit de la découverte même du corps. De la sorte, quoique ménageant un possible effet de suspense, l'ensemble n'ouvre pas sur une réelle attente, et semble déjà voué à l'oubli, car si l'on en croit Laetitia Gonon, «aussitôt lu, s'il n'est pas rappelé dans les éditions suivantes qui le montent en "affaire", le fait divers est aussitôt oublié, car remplacé par d'autres bien souvent écrits comme lui ${ }^{8}$ ». Par ailleurs, l'emploi du déterminant indéfini, qui ne présuppose pas le référent connu, confirme à la fois l'immanence du récit et le statut ordinaire de son personnage principal: le fait divers criminel suscite l'impression que tout un chacun aurait pu en être la victime.

Mais on trouve dans les pièces des exemplifications plus outrées encore, qui font fi de toute crédibilité pour mieux jouer avec les codes du genre. La légère surprise née du sentiment d'absurdité dans La Scène (le corps a été découvert à Berck-Plage, l'enquête est en cours à Limoges) laisse place à un trouble plus marqué dans Le Vrai Sang, lorsque La Machine à dire beaucoup se cite elle-même :

La machine à dire beaucoup communique: «Un Quidam surnommé Quelqu'un, auquel seraient venus se joindre deux individus sans alibi supposés de la même farine, se sont rassemblés ce matin devant l'Assemblée du Syntagme avec un tiers de la même espèce. Et ce, pour former le premier comité quadripartite destiné à conforter dans leur opinion les sondés sans opinion. » (VS, p. 45)

Il n'est plus question de produire un fait divers recevable, mais bien de mettre en évidence - pour le tourner en dérision - une caractéristique mentionnée plus haut, qui a partie liée à l'anonymat. Le pronom acquiert ici certains traits du nom propre, tout en conservant une structure proche du groupe nominal : dans "un Quidam» comme dans «Quelqu'un », malgré le figement des expressions, la présence de l'article 
indéfini est encore nettement perceptible. Un tel jeu de substitution vise la mise au jour de ce que nous pourrions appeler un patron générique : un modèle du genre.

Cependant, et la chose est manifeste dans les paroles relayées par La Machine à dire beaucoup, ce n'est pas tant le micro-drame qui retient le dramaturge que son traitement médiatique : non l'événement même, mais sa relation. Valère Novarina se saisit d'une matière verbale, c'est-à-dire du fait divers déjà institué comme fait divers. Dans La Scène, ce phénomène est d'autant plus net que le spectateur est confronté à la présence physique, matérielle, du journal papier sur la scène, dans les mains du personnage qui a pour nom Tête d'Isaï ${ }^{10}$. Dans Le Vrai Sang, on assiste à un usage croissant des guillemets, qui renforce l'effet citationnel, et invite à penser l'information moins en termes de production que de reprise, de répétition, de circulation. À cela s'ajoute le recours, déjà initié en 2003 mais systématisé en 2011, à la formule inaugurale «La Machine... communique:», qui présente toute parole comme déjà médiatisée. Ainsi, une Machine peut citer une autre Machine, mais peut aussi se citer elle-même : c'est le cas dans l'intervention de La Machine à dire beaucoup mentionnée précédemment. Une fois encore, et de façon plus aiguë dans Le Vrai Sang, le fait divers est appréhendé comme une construction discursive.

7 In fine, la distinction entre la matière et sa mise en forme se trouve invalidée : il semble que l'événement ne préexiste pas à sa mise en forme, mais en résulte. Chacune des pièces pousse la logique fait-diversière jusqu'à son comble, prenant acte de l'idée que le genre, "geste des obscurs, [...] est épopée de l'insignifiant ${ }^{11}$ ». Le fait même le plus dérisoire peut devenir divers, une fois intégré à une série d'informations. Ainsi dans $L a$ Scène :

LA MACHINE À DIRE LA SUITE

Un chien poursuivi par zoolâtrie vient d'être arrêté alors qu'il tentait de passer le col d'Aspin ou du Lauraret.

Bruits de bottes à la pédiathèque municipale de Vitry-le-Sec.

Le récidiviste vient de faire machine arrière en lançant des arguments tous azimuts.

(S., p. 97)

8 Ce «bruit de bottes" est institué en fait divers à la faveur d'un jeu de mots ${ }^{12}$ et constitue une forme de provocation à l'égard du lecteur et du spectateur, car par un effet de contamination, le soupçon d'insignifiance est porté sur tous les récits diffusés. On peut également reconnaître ici une allusion aux préparatifs de guerre (l'expression figée «bruit de bottes » relève du domaine militaire), mais la menace que pourrait constituer l'arrivée de troupes semble se dissoudre sous l'effet comique du néologisme. Si une tension sourd malgré tout, c'est donc sur fond d'absurdité, dans le contraste entre une mention anodine et une violence latente. La gageure consiste en définitive à créer un fait divers vidé de tout contenu propositionnel, ou bien dont le contenu propositionnel serait réduit autant que possible, par exemple avec le récit d'un nonévénement :

LA MACHINE À DIRE LA SUITE

On porte à notre connaissance que des faits d'importance de force plus 3 seraient parvenus à notre connaissance la nuit dernière entre $23 \mathrm{~h} 56$ et $23 \mathrm{~h} 8$ - et ne se seraient malheureusement finalement pas produits. ( $S$, p. 11) 


\section{Rouages linguistiques}

9 La réception du fait divers passe donc avant tout par la reconnaissance de traits syntaxiques, lexicaux, énonciatifs, identifiés comme caractéristiques du genre. Valère Novarina se livre, dans les termes de Gérard Genette ${ }^{13}$, à une imitation stylistique dont le registre oscille entre le ludique et le satirique. Le pastiche ou la charge ne visent cependant pas un auteur : le fait divers se présente comme la relation par un quidam de la mésaventure d'un quidam ${ }^{14}$. Mais le prétendu effacement auctorial a paradoxalement pour corollaire un surmarquage linguistique: les pièces pointent ces récurrences, qu'elles accentuent jusqu'à produire un effet de saturation.

Ainsi du recours systématique à des périphrases aspectuelles, lesquelles présentent le procès comme tout juste achevé : « un enfant de trois jours vient d'être découvert [...] » (S, p. 29) ; « une requête en problématique vient d'être lancée $[. .$.$] » (S$, p. 134) ; « [u]n instrument de sondage vient d'être exhumé des ruines du parlement nord-poitevin $[. .] ».(A I$, p. 70$)$. Ce trait, déjà caractéristique du genre au xIX siècle, semble ici renforcé par l'impression de prise directe sur les événements permise par les nouvelles technologies. À l'ère du numérique, l'information surgit en continu et sans délai, de la même manière que surgissent sur scène les différentes machines. Une didascalie annonce, dans La Scène : «La machine à dire la suite, surgissant » (S, p. 69); de façon générale, il semble que les prises de parole mécaniques soient, dans les trois pièces, intempestives. Une prédilection pour la voix passive est par ailleurs nettement perceptible dans ces passages : l'attention est portée sur un seul actant, le complément d'agent n'étant, dans la majorité des cas, pas exprimé (ce phénomène syntaxique, typique des rapports de police, est largement répandu dans l'écriture fait-diversière).

En somme, le fait divers serait un discours a minima: si l'on peut parler de microdrame, c'est que s'y articulent souvent unité de temps, unité de lieu, unité d'action ${ }^{15}$. À ces unités répondraient alors celle, syntaxique, de la phrase articulée autour d'un unique noyau verbal. Pour autant, l'écriture fait-diversière contemporaine telle que la représente Valère Novarina est loin de la concision que l'on pourrait attendre du genre. Si la structure de la phrase simple est conservée, elle se trouve perturbée par l'insertion d'éléments qui compromettent toute aspiration à l'épure. En témoigne la présence de nombreuses constructions détachées, avec notamment des séries d'appositions, qui provoquent un effet de dilatation: "[h]abillé d'un corps appartenant à un autre et revêtu d'apparences, un passager, cité témoin à la barre du Tribunal Impénitent de purification des erreurs, a déversé devant le jury ébahi le contenu d'un troisième larron chuté du toit » (VS, p. 98). L'accumulation de participes présents procède du même élan, qui peut conduire la phrase à la limite de la recevabilité syntaxique : «le policier n'en pouvant plus de ce dernier refusant de répondre » $(S$, p. 135$) ;$ « $[u] n$ veuf ayant décidé de se faire inséminer par son chien mort depuis six mois, n'en pouvant plus le chien luimême ayant été peu auparavant euthanasié, vient de voir prorogée l'annulation de son contrat corporel avec l'animal» (VS, p. 112). Associé à ces procédés, le recours aux pronoms cataphoriques produit un effet de suspens et parfois de suspense :

Éperdument amoureux de toutes sans savoir laquelle, un Ébroïcien de Louviers de vingt-huit printemps a méthodiquement dévoré pendant le dernier mois d'août les cent vingt-quatre mains de ses soixante-deux cousines devant l'œil indifférent de son filleul le petit Cédric, huit ans, qui filmait la scène avec une caméra de location. Loca-image : 0859897633. L'enquête est en cours au cours d'une enquête en activité. (S, p. 70) 
12 Au niveau lexical, les récits sont largement nourris par l'interdiscours, c'est-à-dire par «ce fonds commun d'expressions administratives, policières ou médicales que reprennent les faits divers du siècle ${ }^{16} »$ : la critique étudie le $\mathrm{XIX}^{\mathrm{e}}$ siècle, mais on peut par extension considérer que l'écriture fait-diversière d'une époque puise dans un fonds linguistique qui lui est contemporain. La contamination du discours par le vocabulaire technique emprunté à des champs spécialisés est donc un phénomène sinon définitoire du moins caractéristique du genre. La surprise naît, chez Valère Novarina, du télescopage de plusieurs domaines: « $[u] n$ espace cervical vient d'être aménagé en zone tampon dans la tête du médiologue de la république » (VS, p. 105). On assiste ici à une curieuse superposition géo-médico-politique, qui feint la rigueur pour mieux dénoncer certains emplois abusifs: tout se passe comme si la parole était dupliquée mécaniquement d'un média à l'autre, sans que son sens ne se trouve jamais interrogé.

De cette feinte quête de précision participent aussi les nombreuses indications numérales, qui peuvent assumer diverses valeurs, comme dans ce passage du Vrai Sang:

LA MACHINE À MARCHER À L'ARRÊT.

La haute autorité à comptabiliser la mort communique: "Devant l'agence départementale de la disparition, deux jeunes de la CLAPUD, originaire du six quat'neuf, fumant des P-quatorze et brandissant un gros Huit-trente-trois, ont regagné à la hâte leur TT-huit-turbo et rejoint l'A-trois à hauteur du P.k vingt et un, où, devant une bretelle où ils ont stationné dix minutes dans un sèpte-seven, s'est abattu un agent de tort de la FBC, intitulé par sa maman Paul Clamsydre. Les deux bambins, animaux de souche, ont été replacés en temps voulu en zone de bien sécurisée, puis remis sous huis clos aux autorités des organisations non parentales compétentes. (VS, p. 49-50)

Le comique repose en partie sur le glissement inattendu d'un code sémiotique à l'autre, en l'occurrence des chiffres arabes aux lettres latines, glissement qui procure un sentiment d'étrangeté (la familière autoroute A3 est moins aisément reconnaissable sous le nom d'A-trois, quant au sèpte-seven, qui met en coprésence deux langues, il pourrait bien s'agir d'une variation facétieuse de célèbres enseignes de supermarchés, dont les noms - 7-Eleven, 8 à Huit - mêlent différents systèmes de notation). La présence de fautes d'orthographe renforce le sentiment de confusion : ici il est question de « deux jeunes de la CLAPUD, originaire du six quat'neuf, [...] »), ailleurs du « Tribunal Internationnal Communicatoire » (VS, p. 100) ou encore de "camérahmanes arbitrant la vindicte » (ibidem). On peut trouver là, sans nul doute, un écho à la multiplication des coquilles dans les journaux, ainsi qu'à la prévalence de la langue anglaise (dont le « sèpte-seven » donnait déjà un exemple).

Il semble que Le Vrai Sang conduise à son comble une logique de déstabilisation initiée dans les autres pièces : non seulement la mécanique fait-diversière occupe une place sans précédent, mais elle est aussi plus marquée, de sorte que la veine satirique s'impose nettement. Les sigles et acronymes ininterprétables ou improbables (FBC et CLAPUD dans La Scène), acquièrent des dimensions nouvelles dans la dernière pièce, avec par exemple la mention du THOVRSSL-DPPCINVV : Très Haut Observatoire à la Virtualité du Réel Sous Surveillance Latérale et de Détection de la Présence de Précarités Concrètes Imputables à la Non-Visibilité du Visible (VS, p.111). L'unité lexicale semble soumise à un mouvement d'expansion potentiellement infini - cet excès est précisément contraire à la logique qui régit d'ordinaire l'emploi des siglaisons, à savoir le souci d'économie. Par ailleurs, toutes les pièces sont le lieu d'un 
jeu avec le nom propre : il s'agit là encore de pointer, en le caricaturant, un usage abusif du discours médiatique en général, et de l'écriture fait-diversière en particulier, qui élève un quidam au rang de protagoniste, non sans une certaine complaisance dans le récit de ses déboires. La présence de formules volontairement lourdes et maladroites provoque une forme de défamiliarisation. C'est le cas dans une construction détachée telle que "intitulé par sa maman Paul Clamsydre ", apposée à " un agent de tort de la $\mathrm{FBC} »(V S$, p. 49) : l'expression est doublement étrange, parce qu'elle associe des termes inappropriés (le participe passé intitulé peut difficilement s'appliquer à un humain) et le passage brutal à un registre de langue familier et enfantin, tout à fait inattendu dans le champ journalistique (avec le substantif maman).

Enfin, le recours à des formules figées conclusives ou introductives redondantes ou tautologiques dénonce, outre l'automatisme, une certaine vacuité. La présence de phrases telles que "L'enquête est en cours au cours d'une enquête en activité » ( $S$, p. 70) ou "Notre enquête de nos enquêteurs » (ibidem) n'est de toute évidence pas justifiée par le contenu propositionnel : il s'agit plutôt de baliser le discours ou, plus encore, de le nourrir artificiellement. Ce qui se présente comme le langage de la communication relève du bruit plus que de la parole : privé de souffle, il est aussi privé de matière. De même, si la Machine dit beaucoup, c'est peut-être précisément parce qu'elle ne dit rien. Encore faut-il s'entendre sur ce rien - ou mieux, entendre ce rien, pour en renverser le pouvoir paralysant.

\section{Opacification : les mots comme des choses}

17 C'est sans aucun doute dans Le Vrai Sang que le phénomène de bascule du trop-plein vers le vide se manifeste de façon la plus évidente. La parole mécanique apparait toujours, nous l'avons vu, comme une reprise, de sorte que se met en place, fictivement, un large réseau intertextuel, chaque machine en citant une autre ou se citant ellemême, dans un jeu de renvoi potentiellement infini. Mais il arrive que, par un emballement de ce processus, l'élément cité disparaisse tout à fait :

LA MACHINE MÉTRIQUE
L'Entité beauceronne, le Front de Libération du Rouergue, la prétendue Autorité
morvandiotte, le collectif Combien ça coûte, la Société de Reproduction et de
Multiplication du Réel, la société de production de l'Émission Main mise, la société
de production du Cycle J'y suis j'y reste, la société de production de l'émission de la
rediffusion de Pas la peine d'en parler, la société d'élaboration laborieuse d'Y a rien à
foutre, la Haute Mission à la société de reproduction de l'émission Société fermière,
l'Union pentadrillheptique ainsi que l'Agence nationale à l'ingérence locale dans la
gouvernance mondiale communiquent: .... (VS, p. 103)

18 Les points de suspension signalent typographiquement une béance et peuvent engager une double interprétation: d'une part, seul importe le fait de communiquer (il n'est donc pas nécessaire, et il est peut-être même superflu, de restituer ce qui est communiqué), d'autre part, il n'est pas même certain que quelque chose puisse être communiqué, au-delà de la valeur pragmatique accordée à la prise de parole (de l'énoncé ne demeurerait donc que la force illocutoire, pour reprendre la partition établie par Austin ${ }^{17}$ ). Cet enrayement est une invitation à faire retour sur nos propres pratiques discursives.

19 La question du langage est absolument centrale dans le théâtre novarinien, et fait par ailleurs l'objet de plusieurs développements dans des textes en prose qui relèvent 
plutôt de l'écriture essayiste ou aphoristique, tels que Devant la parole $e^{18}$ ou Lumières $d u$ corps $^{19}$. Un glissement est sensible dans nos pièces, puisque les mots s'imposent peu à peu comme objets des nouvelles relayées :

LA MACHINE LINÉAIRE.

La Société Sociétale des Cohésions Obliques communique : « Deux lévriers viennent d'être déclarés contre-sujets par trois présumés dogues circulaires ; ils se sont croisés sans un mot: croisant leurs reflets comme des nomades dans un miroir, pour reprendre l'heureuse formule du poète Marie-Godfroy Loubet. »

La Machine à en dire plus.

L'Agence de l'égalité des chances entre grands et perdants du visible communique :

«Un animal se passant de son propre langage va venir.» L'ensemble, faisant sens, resens, et même faisant même rythme. (VS, p. 64)

De façon très marquée ici, plus ténue ailleurs, le dire se double d'un mouvement réflexif qui nous pousse à interroger les conditions mêmes de son apparition. Ce dernier passage nous semble d'ailleurs doté d'une valeur heuristique, puisqu'il fait du rythme la pierre de touche de toute réflexion linguistique. Valère Novarina se donne explicitement pour tâche, dans le prologue du Vrai Sang, de «[t]émoigner des maladies du langage » (VS, p. 17) : au premier rang de celles-ci, l'absence de déploiement rythmique, autrement dit, l'imposition d'une logique binaire, manichéenne, qui prive le mot de toute sa force de résonance. Dès 2003, on rencontre des enrayements sinon semblables du moins homologues à celui déjà mentionné :

LA MACHINE À DIRE LA SUITE

[...] Un Sédunois de mille ans et un Sparnassien de la même farine. - Avant de boire d'un trait ce pot de démence virale et liberticide. - Versant ainsi une eau précieuse au moulin des émules de la pensée adverse. - Et ce, sans vouloir offenser nos voisins d'outre-Venoge, ni d'outre-Quiévrain, ni d'outre-Loire, ni d'outre-Bidassoa. - Inféodé. - Zone tampon. - Payé rubis sur l'ongle. - Battant la chamade. Inféodé aux succubes de la zone tampon. (S, p. 149-150)

21 L'emballement et la fragmentation sont les symptômes de l'atrophie de la matière verbale, dès lors que celle-ci est instrumentalisée. Les constructions détachées restent en suspens, dans l'attente d'un référent stable, ce qui provoque un effet de flottement syntaxique généralisé. En 2007, les points de suspension se substituent au tiret, accentuant l'impression de perte, de délitement :

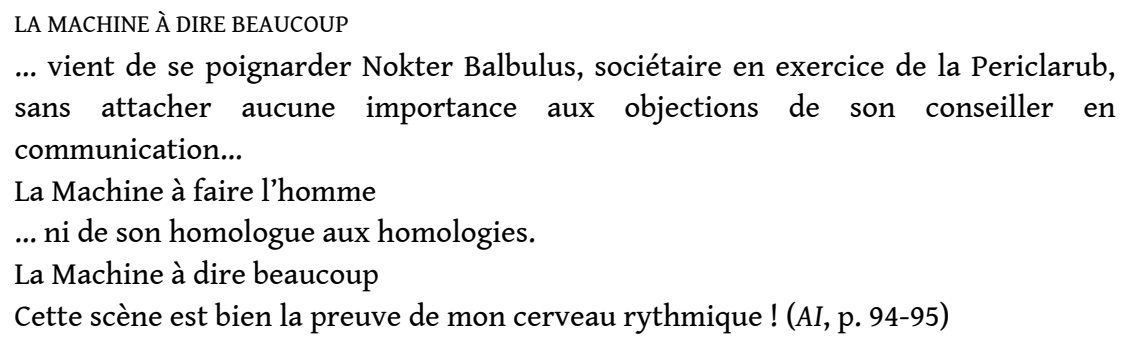

Le commentaire de La Machine à dire beaucoup, parce qu'il interrompt la froide dynamique d'accumulation, opère une bascule d'ordre métatextuel : l'enjeu de la scène est bien de renverser le rythme mécanique, désincarné et asséchant propre aux médias. On trouve un écho direct de cette proposition dans Lumières du corps :

129. Chaque jour, autour de nous, le langage perd un peu plus son volume et la pensée sa respiration; les détours respirés, les chemins de traverse, les courbes sont interdites : tout va vers le linéaire, la phrase à sept, cinq, trois mots, le slogan ; tout va vers une langue plate, uniforme, répétitive, incapable de restituer le drame de la pensée, de développer ses volutes contradictoires. Par l'expansion universelle d'une pensée binaire, un rythme à deux temps, le manichéisme se répand et gagne 
tout. Comme si notre pensée aujourd'hui - et le langage humain - avait le souffle coupé ; comme si respirer, aller au bout de la phrase, traverser la noyade, renverser les mots, retourner les sens, brûler le langage par notre corps et s'y perdre, nous était interdit. Tout doit être de surface, suréclairé, sans ombre aucune, sans volume, présenté sous son meilleur jour et toujours à vendre: avec étiquette, mode d'emploi, prix et résumé du contenu... (LC, p. 73-74)

Une circulation s'établit entre l'exploration théâtrale et celle, plus proche de l'essai, des textes réflexifs, sans que l'on puisse établir entre eux de préséance, ni de partition claire entre ce qui relèverait du théorique et ce qui relèverait du pratique : on parlera plutôt de prolongements réciproques, dans la mesure où chaque genre offre sa propre réponse en acte, c'est-à-dire en mots, à l'«ingurgitation communicationnelle » ("Prologue», VS, p. 19). En ce sens, on ne saurait voir dans les pièces une pure entreprise de dérision. Pastichant l'écriture fait-diversière, le dramaturge ne se contente pas d'en révéler les structures répétitives ni d'en dénoncer la vacuité : son geste ne relève pas de la seule charge, mais ouvre au contraire le champ des possibles rythmiques, par le jeu. En premier lieu, un renversement est opéré par la surprise qui ne manque pas de saisir le lecteur comme le spectateur : les détournements inattendus, les tours de passe-passe syntaxiques ou lexicaux (ainsi du glissement catégoriel précédemment décrit, qui attribue à Quidam certains traits du nom propre) ou la brusque infraction à certains invariants génériques désamorcent l'effet asphyxiant, compromettent la linéarité. C'est le cas lorsque le discours non-embrayé accueille soudain la première ou la deuxième personne :

LA MACHINE À PASSER À L'ARRÊT

«Deux Carcassonnais de profil, l'un pourvu d'une troisième tête en trop, ont mangé, selon témoin, deux boîtes de biscuits-spontanés devant treize de nos animaux, nos camérahmanes arbitrant la vindicte... La parole sera-t-elle redonnée à ces sympathiques quadrupèdes ?... Jean-Laurent Blossu, leur avez-vous prêté mainforte? » Dites pourquoi! Pourquoi ? Pourquoi ? (VS, p. 101)

Le caractère grotesque de la situation décrite, la prise à partie de l'interlocuteur, l'implication finale de l'énonciateur, qui laisse sourdre une angoisse (relative, une fois encore, au dire) réintroduisent précisément le souffle manquant; il n'est pas impossible ici de considérer qu'on passe d'un premier modèle, celui du journalisme écrit, à un second, celui du reportage télévisé. Mais la dimension pneumatique est aussi affaire de corps, de voix, de respiration : à ce titre, la mise en scène, au sens littéral du terme, engage une circulation qui est tout à fait étrangère aux médias. En somme, le passage d'un médium à un autre engage une nouvelle réception, intimement liée, en l'occurrence, à l'espace physique du théâtre. Il s'agit pour le dramaturge et les acteurs de donner à percevoir le langage comme "matière vivante » et « champ de force » $(D$, p. 21). D'où une conception quasiment organique des mots, qui cessent d'être considérés comme instruments de description des choses du monde pour être appréhendés eux-mêmes comme objets du monde: «[l]es mots vont dans l'espace comme des objets qui s'ouvrent. Les mots sont des logaèdres » (D, p. 22). Cette attention portée au déploiement physique de la parole peut être mise en relation avec le phénomène énonciatif que Jacqueline Authier-Revuz appréhende en termes d'opacification et de non-coïncidence. Il est d'ailleurs frappant de constater à quel point l'analyse proposée par la linguiste entre en résonance avec la conception que développe Valère Novarina :

Dans tout fait de réflexivité autonymique, il y a un signe qui s'impose comme objet, propulsé sur le devant de la scène comme "personnage» auquel le dire fait 
référence, sortant par là de son rôle de rouage ordinaire de la machinerie du dire, voué à l'effacement dans l'accomplissement de sa fonction ordinaire de communication $^{20}$. dans lequel il se trouve pris pour le propulser, au sens littéral, «sur le devant de la scène ». Les structures étudiées par Jacqueline Authier-Revuz relèvent du fait autonymique, c'est-à-dire de la représentation de la non-coïncidence. Nous élargissons pour notre part le constat, et parlons ici du phénomène d'opacification en un sens large, intégrant la manifestation de la non-coïncidence. Il y a représentation lorsqu'un jeu de mots occasionnel se trouve doublé par une marque de type méta-énonciatif, comme dans l'exemple précédemment cité : «Pour reprendre l'heureuse formule du poète Marie-Godfroy Loubet. » La manifestation, en revanche, consiste à donner à voir ou à entendre la non-coïncidence sans toutefois en proposer de commentaire, qu'il soit phrastique ou typographique: c'est le cas avec l'emploi de néologismes («l'Union pentadrillheptique ») ou encore avec les perturbations graphiques (« camérahmanes »). propriétés éminemment charnelles, propriétés que nie ou occulte le traitement de l'information non seulement dans le fait divers, mais dans tout le champ journalistique. Le fait divers s'inscrit ainsi dans un réseau plus large, qui inclut les bulletins d'information, les bulletins météorologiques, les slogans politiques et publicitaires. Les frontières entre les différents genres de discours médiatiques sont parfois poreuses - que l'on pense à certaines scènes qui, dans les trois pièces, ménagent un effet de confusion généralisée, par télescopage ou contagion : ainsi du «Ballet mécanique » en 2003 (S, deuxième scène de «L'Acte théorique »), du « Charivari » en 2007 (AI, II, 10) ou encore des « Vingt-neuf machines au point mort » en 2011 (VS, I, 11). Nous avons déjà rencontré un exemple on ne peut plus frappant avec l'intégration d'un numéro de téléphone au sein du fait divers, le récit du crime devenant prétexte à la promotion publicitaire (« huit ans, qui filmait la scène avec une caméra de location. Loca-image : $0859897633 »)$.

« Nous finirons un jour muets à force de communiquer » : ce constat établi dans Devant la parole (p. 13) n'est qu'en apparence paradoxal, puisqu'on est amené à dissimiler, avec l'auteur, l'activité de parler et celle de communiquer. On ne saurait cependant établir entre les deux une opposition binaire, ni voir dans le projet dramaturgique une tentative de pure substitution. Il s'agirait plutôt pour Valère Novarina - et là se trouve la gageure - de réinstaller la parole dans le lieu qui semblait l'en exclure, à savoir celui de la communication. Le renversement s'opère à l'intérieur même du discours médiatique : plus qu'une simple mise en cause de la production mécanique, les pièces sont une invitation à installer le " développement soufflé » ("Prologue», VS, p. 19) au cœur de ce qui, par définition, en paraissait privé. Autrement dit, l'investissement dramaturgique de l'écriture fait-diversière, en pointant les apories de la "Logologie " (LC, p. 71), ouvre pour les mots un espace de résonance, par un triple jeu : jeu de reprise et de détournement des codes du genre, jeu linguistique et jeu de l'acteur, dont la présence physique sur scène compromet le rythme des machines, tout en le mimant. Le travail d'opacification nourrit ainsi une conception chorégraphique du langage, dont d'auteur expose les linéaments dans Lumières du corps :

Qu'est-ce que les mots nous disent à l'intérieur où ils résonnent? Qu'ils ne sont ni des instruments qui se troquent, ni des outils qu'on prend et qui se jettent, mais qu'ils ont leur mot à dire. Ils en savent sur le langage beaucoup plus que nous. Ils 
savent qu'ils sont échangés entre les hommes non comme des formules et des slogans mais comme des offrandes et des danses mystérieuses. (LC, p. 15)

\section{NOTES}

1. V. Novarina, La Scène, Paris, P.O.L., 2003, p. 134 (désormais S).

2. V. Novarina, L'Acte inconnu, Paris, P.O.L., 2007 (désormais AI).

3. V. Novarina, Le Vrai Sang, Paris, P.O.L., 2011 (désormais VS).

4. R. Barthes, "Structure du fait divers" [1964], dans Essais critiques, Euvres complètes, t. II, É. Marty (éd.), Paris, Seuil, 2002, p. 442-451.

5. L. Gonon, Le Fait divers criminel dans la presse quotidienne française $d u X_{X} x^{e}$ siècle : enjeux stylistiques et littéraires d'un exemple de circulation des discours, thèse de doctorat en sciences du langage, dir. G. Philippe, Université Sorbonne Nouvelle - Paris 3, 2011, p. 34.

6. M. M'Sili, Le Fait divers en République: histoire sociale de 1870 à nos jours, Paris, CNRS Éditions, 2000, p. 87.

7. Ibid., p. 95.

8. L. Gonon, Le Fait divers criminel dans la presse quotidienne française du XIX ${ }^{e}$ siècle, ouvr. cité, p. 34.

9. C. Deleu, qui compare différentes définitions, note d'ailleurs bien que le fait divers est un objet médiatique : une phrase telle que "Il m'est arrivé un fait divers!" paraît irrecevable (C. Deleu, «Introduction », dans Les Cahiers du journalisme, $\mathrm{n}^{\circ}$ 14, printemps-été 2005, p. 10).

10. Une didascalie précise : «dans le quotidien » $(S$, p. 33), ce qui suggère que Tête d'Isaïe et Tête de Rachel lisent les informations. Le fait surprenant est qu'elles lisent les informations à venir, de sorte que la partition entre passé et futur devient poreuse.

11. M. Perrot, Les Ombres de l'Histoire. Crime et châtiment au XIX siècle, Paris, Flammarion, 2001, p. 280.

12. Le substantif bottes entraîne l'élément pédo-, en rapport au sol que foulent les bottes, et proche de podo-, qui lui concerne le pied - ce morphoème provoquant l'altération de l'initiale de médiathèque.

13. G. Genette, Palimpsestes : la littérature au second degré, Paris, Seuil, 1982.

14. Il est vrai que l'écriture fait-diversière quitte peu à peu l'anonymat au xIx siècle, mais le nom des auteurs indiqué au bas de la rubrique est volontiers générique, et parfois inventé.

15. M. M'Sili, Le Fait divers en République, ouvr. cité, p. 96.

16. L. Gonon, ouvr. cité, p. 279.

17. J. L. Austin. Quand dire, c'est faire [1962], Gilles Lane (trad.), Paris, Seuil, 1970.

18. V. Novarina, Devant la parole, Paris, P.O.L., 1999 (désormais D).

19. Le quarante-et-unième aphorisme de Lumières du corps balise d'ailleurs très clairement cet enjeu : «41. En ce temps de communication galopante, de manie communicante, de publicité et de propagande perpétuelle, d'humanisme forcé, tout ce qui touche au langage est au centre, agissant et politique. Être à l'œuvre dans le langage, être son explorateur, son gardien, c'est être au cœur même des forces qui nous opèrent aujourd'hui » (V. Novarina, Lumières du corps, Paris, P.O.L., 2006, p. 67 [désormais $L C]$ ).

20. J. Authier-Revuz, « Le fait autonymique : langage, langue, discours. Quelques repères », dans J. Authier-Revuz, M. Soury et S. Reboul Touré (dir.), Parler des mots : le fait autonymique en discours, Paris, Presses Sorbonne Nouvelle, 2003, p. 71. 


\section{RÉSUMÉS}

L'article s'intéresse à l'écriture du fait divers dans trois pièces de Valère Novarina: La Scène (2003), L'Acte inconnu (2007) et Le Vrai Sang (2011). Chacune de ces pièces est le lieu d'un jeu avec les codes du genre fait-diversier, nettement perceptible dans les interventions de « La Machine à dire beaucoup ", personnage qui apparait comme un relais du discours journalistique. Notre étude s'attache à mettre en évidence, par une approche lexicale, syntaxique et énonciative, ce que la reprise subversive de V. Novarina entend révéler du fait divers, et de son traitement médiatique contemporain. In fine, il s'agit pour le dramaturge de renverser, par un effet comique de surcharge, la paralysie et l'asphyxie de "l'empire de la Communication », afin de substituer au discours médiatique désincarné une exploration sensible des possibles du verbe.

This paper addresses the writing of crime news items in three theatre plays by Valère Novarina : La Scène (2003), L'Acte inconnu (2007) and Le Vrai Sang (2011). Each of these plays questions the genre codes, as clearly seen when "La Machine à dire beaucoup", a character personifying the media language, appears on stage. Our point is to analyse, through a lexical, syntactic and enunciative approach, what the subversive appropriation of Novarina reveals of the values placed on the crime news genre in our contemporary world. Ultimately, the playwright aims to reverse, through comical excess, the paralysis and the asphyxia induced by the "empire of Communication", so as to replace the disembodied media discourse by a physical exploration of language.

\section{AUTEUR}

\section{SIBYLLE ORLANDI}

Université Statale (Milan), Institut français Italia.

Docteure qualifiée en langue et littérature françaises et en Sciences du langage, agrégée de Lettres modernes et ancienne étudiante à l'ENS de Lyon, Sibylle Orlandi est actuellement lectrice à l'Université Statale de Milan et à l'Institut français Italia. Elle a soutenu en 2015, à l'Université Lyon 2 Lumière, une thèse consacrée aux créations poétiques et plastiques de Ghérasim Luca et poursuit aujourd'hui des recherches en stylistique dans le champ de la littérature des $\mathrm{Xx}^{\mathrm{e}}$ et $\mathrm{XxI} \mathrm{e}^{\mathrm{e}}$ siècles. 